УДК 515.124.4

\author{
V. I. KuZ'Mich, A. G. SAVChenKo
}

\title{
GEOMETRIC RELATIONS IN AN ARBITRARY METRIC SPACE
}

\begin{abstract}
V. I. Kuz'mich, A. G. Savchenko. Geometric relations in an arbitrary metric space, Mat. Stud. 52 (2019), 86-95.

The paper is devoted to individual elements of metric geometry. Analytical relations are considered that concern the distances between points of a metric space and have a definite geometric interpretation. These relations are established on the basis of the concept of angle as an ordered triple of points of a metric space, its numerical characteristic, and are carried out in Euclidean geometry. It is shown that these relations are satisfied for points of an arbitrary metric space.
\end{abstract}

1. Introduction. The paper is devoted to certain questions of metric space geometrization. In a number of papers [1-5] the question of applying the notion of an angle which is formed by three points of a metric space as an ordered triple of these points and his numerical characteristic (angular characteristic), was studied. This allowed us to use an analytical approach to the study of such concepts as straight-line placement and planar placement of points in a metric space. It should be noted that these studies have been carried out without the need for the completeness of space, its linearity, dimension, and without any restrictions on the metric space, and can be used in metric spaces with finite or countably many points. This approach, in our opinion, enables to conduct a discrete geometric structuring of the metric space, introducing into its consideration habitual geometric images and concepts of the Euclidean geometry.

The concept of the straight-line placement of points of a metric space was studied in detail by V. F. Kagan [6, sect. XIX; 7, p. 527]. V. F. Kagan gave an axiomatic definition of a straight line, establishing isomorphism of the set of real numbers and the set of points of a metric space satisfying 17 postulates divided into 4 groups. In [5, p. 386] the set $L_{I}$ of points of an arbitrary metric space is constructed, satisfying the first group of these postulates, which contains five placement postulates. Since the other postulates (structures, congruence, continuity, and unboundedness) were not used, this made it possible to apply the results to finite or countable metric spaces. In addition, it became possible to introduce the concept of angle, and its numerical characteristic (angular characteristic), which are not related to the completeness or linearity of a metric space. In turn, the possibility of a logical generalization of the notion of straight-linearly placement of points by introducing the concept of planar placement of points of a metric space has appeared. Moreover, both these concepts, and their relationship, are fully described using the angular characteristics [5, p. 387].

2010 Mathematics Subject Classification: 51F99.

Keywords: metric space; angle in the metric space; straight-linearly placement of points of metric space; straight-linearly ordered placement of points of metric space; planar placement of points of metric space. doi:10.30970/ms.52.1.76-85 
It should be noted that, despite its importance, the concept of the angle, and its dimensions, are still quite ambiguous in nature. Euclid [8, p. 11-12] describes the angle using its five properties, defining as follows: "a planar angle is the inclination of two lines to each other, meeting in plane with each other, but not placed along (one) straight line". Heron defined the angle as "compressing the surface by broken line at a point" [8, p. 230]. Further, the angle was defined as "direction deviation during its rotation" [8, p. 231], "the indefinite part of the plane bounded by two lines that intersect at one point" [8, p. 231], "a figure formed by two half-straight lines emerging from the same point" [9, p. 26]. D. Hilbert called the angle " $a$ system of two rays emanating from one point in the plane and belonging to different straight lines" [10, p. 10].

For modern metric geometry, abstraction from the concept of angle as a figure is characteristic. In fact, numerical characteristic of the angle is also called angle [11, p. 36; 12, p. 96; 13, p. 212]. This in our opinion somewhat separates the concept of angle from its intuitive (classical) understanding. In addition, the concept of angle is introduced only for Euclidean spaces, which makes it very difficult and insufficiently substantiated to apply the obtained results to nonlinear, finite, or countable metric spaces.

The question arises whether there are such analytical relations between points of a metric space that hold in Euclidean geometry, but also valid in any metric space, regardless of the choice of the metric? And if so, what determines these ratios? Below it will be shown that a significant part of the known geometric relations can be obtained based on the concept of angular characteristics obtained from the classical cosine formula. Such an approach in metric geometry was proposed by A. D. Aleksandrov [11, p. 36].

2. Preliminaries. In the following, all points of the metric space $(X, \rho)$ will be considered distinct, that is, we will consider only the positive values of the metric $\rho$ of the space $X$. The set of three points $x_{1}, x_{2}, x_{3}$ of the space will be called a triangle, and denoted by $\Delta\left(x_{1}, x_{2}, x_{3}\right)$. In this case, the points themselves will be called vertices, and the pairs of points $\left(x_{1}, x_{2}\right)$, $\left(x_{1}, x_{3}\right),\left(x_{2}, x_{3}\right)$ the sides of the triangle. In this paper, we will use the concept of an angle formed by three points of a metric space [1, p. 28].

Definition 1. Let $x_{1}, x_{2}, x_{3}$ be arbitrary points of a metric space $(X, \rho)$. An ordered triple $\left(x_{1}, x_{2}, x_{3}\right)$ of these points will be called an angle with a vertex at the point $x_{2}$ and denoted by: $\angle\left(x_{1}, x_{2}, x_{3}\right)$. The couples of points $\left(x_{1}, x_{2}\right)$ and $\left(x_{2}, x_{3}\right)$, in this case, will be called the sides of the angle.

To compare the angles we will use the concept of angular characteristics $[1$, p. 29; 11 , p. $36 ; 12$, p. 96-97].

Definition 2. Let $x_{1}, x_{2}, x_{3}$ be arbitrary points of a metric space $(X, \rho)$. We will call the real number

$$
\varphi\left(x_{1}, x_{2}, x_{3}\right)=\frac{\rho^{2}\left(x_{1}, x_{2}\right)+\rho^{2}\left(x_{2}, x_{3}\right)-\rho^{2}\left(x_{1}, x_{3}\right)}{2 \rho\left(x_{1}, x_{2}\right) \rho\left(x_{2}, x_{3}\right)},
$$

the characteristic of the angle $\angle\left(x_{1}, x_{2}, x_{3}\right)$, or the angular characteristic.

A metric space $(X, \rho)$ in which the notion of angle is given by Definition 1 , and its characteristic by Definition 2, will be called a metric space with an angular characteristic, and will be denoted by $\Pi$.

In the following, we will use more compact notation of the distance between points: $\rho\left(x_{i}, x_{j}\right)=\rho_{i j}$, and the angular characteristic in the space: 


$$
\frac{\rho_{i j}^{2}+\rho_{j k}^{2}-\rho_{i k}^{2}}{2 \rho_{i j} \rho_{j k}}=\varphi_{i j k} \quad(i, j, k=1,2,3, \ldots) .
$$

In the case of equality $\varphi_{123}=1$, the angle $\angle\left(x_{1}, x_{2}, x_{3}\right)$ will be called a "zero angle", in the case of equality $\varphi_{123}=-1$ a "straight angle", and in the case of equality $\varphi_{123}=0$ a "straight angle".

We will say that points $x_{i}, x_{j}, x_{k}$ are "straight-linearly placement" in the space $\Pi$ if the equality $\varphi_{i j k}^{2}=1$ holds for at least one of these points (for example, for the point $x_{j}$ ), as a vertice of the angle $\angle\left(x_{i}, x_{j}, x_{k}\right)$. In addition, if $\varphi_{123}=-1$, we will say that the point $x_{2}$ lies between the points $x_{1}$ and $x_{3}$, and we call it internal for the points $x_{1}, x_{2}, x_{3}$, and in the case of equality $\varphi_{123}=1$ we will say that the point $x_{2}$ lies outside the points $x_{1}$ and $x_{3}$, and call it extreme for points $x_{1}, x_{2}, x_{3}$.

We will say that the set of points of the space $\Pi$ is a straight-linearly placement if any three points of this set are straight-linearly placement in the space $\Pi$ [5, p. 384].

Formula (1) of the angular characteristic given in Definition 2 is an analogue of the cosine theorem for an arbitrary triangle in Euclidean geometry. Using this definition, it is possible to establish a number of relations for the distances between points of a metric space. These relations are established without any restrictions on the metric of space and carry a certain geometric meaning in the case of a Euclidean space. An example of such a relation is the following statement $[5$, p. 385 , Theorem 1$]$ :

Theorem 1. For arbitrary three points $x_{1}, x_{2}, x_{3}$ of the space $\Pi$, the equality

$$
\left|\begin{array}{ccc}
1 & \varphi_{213} & -\varphi_{123} \\
\varphi_{213} & 1 & \varphi_{132} \\
-\varphi_{123} & \varphi_{132} & 1
\end{array}\right|=1-2 \varphi_{213} \varphi_{123} \varphi_{132}-\varphi_{213}^{2}-\varphi_{123}^{2}-\varphi_{132}^{2}=0
$$

holds.

It follows from Theorem 1 that equality (2) is a necessary condition for the angles of the triangle $\Delta\left(x_{1}, x_{2}, x_{3}\right)$ to have given angular characteristics. This also applies to the "degenerate" triangle, that is, when the points $x_{1}, x_{2}, x_{3}$ are straight-linearly placement in the space $\Pi$. Since equality (2) is obtained for points of an arbitrary metric space, it does not depend on the choice of the space metric.

It follows from equality (2) that in the space $\Pi$ any triangle cannot have more than one straight angle. Indeed, if we assume that all three corners are straight angles in the triangle $\triangle\left(x_{1}, x_{2}, x_{3}\right)$, that is, the equalities $\varphi_{213}=\varphi_{123}=\varphi_{132}=0$ are fulfilled, then equality (2) will not hold, and therefore a triangle with such angular characteristics in space $\Pi$ does not exist.

Now suppose that in the triangle $\triangle\left(x_{1}, x_{2}, x_{3}\right)$ there are two straight angles. Let, for example, $\varphi_{213}=\varphi_{123}=0$. Equality (2) implies that in this case either $1-\varphi_{132}^{2}=0$ or $\varphi_{132}^{2}=1$. This means that the points $x_{1}, x_{2}, x_{3}$ are straight-linearly placement in the space $\Pi$. But in this case $\varphi_{213}^{2}=\varphi_{123}^{2}=1$ [5, p. 385, Theorem 2], which contradicts the assumption made.

The straight-linearly placement of points of the space $\Pi$ is ambiguous compared to the Euclidean geometry. In particular, if the points $x_{1}, x_{2}, x_{3}$ are straight-linearly placement and the points $x_{1}, x_{2}, x_{4}$ are also straight-linearly placement in the space $\Pi$, then their union, the points $x_{1}, x_{2}, x_{3}, x_{4}$, not necessary are straight-linearly placement in this space [3, p. 60-61]. 
Another feature of the points which are straight-line placement in the space $\Pi$ is that the straight-line placement of a finite number of points in space $\Pi$ does not always ensure the existence of "external" points among them.

Example 1. Consider the metric space $R_{0}^{2}$ of ordered pairs of two real numbers $x\left(x_{1}, x_{2}\right)$, the distance between points $x\left(x_{1}, x_{2}\right)$ and $y\left(y_{1}, y_{2}\right)$ of which is defined by the formula

$$
\rho(x, y)=\max \left(\left|x_{1}-y_{1}\right|,\left|x_{2}-y_{2}\right|\right) .
$$

In the space $R_{0}^{2}$ take four points: $a(1,0), b(0,1), c(-1,0), d(0,-1)$, and find the distances between these points

$$
\rho(a, b)=1, \rho(a, c)=2, \rho(a, d)=1, \rho(b, c)=1, \rho(b, d)=2, \rho(c, d)=1 .
$$

According to (1) we find all possible angular characteristics for these points

$$
\begin{gathered}
\varphi(b, a, c)=1, \varphi(b, a, d)=-1, \varphi(c, a, d)=1, \varphi(a, b, c)=-1, \\
\varphi(a, b, d)=1, \varphi(c, b, d)=1, \varphi(a, c, b)=1, \varphi(a, c, d)=1, \\
\varphi(b, c, d)=-1, \varphi(a, d, b)=1, \varphi(a, d, c)=-1, \varphi(b, d, c)=1 .
\end{gathered}
$$

Since the square of each of the angular characteristics is equal to unity, the points $a, b, c, d$ are straight-line placement in the space $R_{0}^{2}$. However, there are no external points among them, since each of them, like the vertex of the angle, has one of the angular characteristics equal to -1 , and therefore lies between some of these points.

The existence of external points among the four straight-line placement ones ensures the condition of their straight-linearly ordering. This concept was introduced in [5, p. 386]. It is proved that the necessary and sufficient condition for the straight-linearly ordering of four points $x_{1}, x_{2}, x_{3}, x_{4}$ of space $\Pi$ is $\varphi_{213} \varphi_{214} \varphi_{314}=1$, at least for one of these points (for points $x_{1}$, for example, as vertices of the corner). For points considered in Example 1, this condition is not satisfied for none of them.

Another feature of the straight-linearly placement of points in the space $\Pi$ is the ratio between angles which are adjacent. In Euclidean geometry, the adjacent angle complements the specified angle to the straight angle. In particular, the adjacent angle for the straight angle is necessarily a straight angle. In the space such a relation is not always fulfilled ([3, p. $66 ; 5$, p. 388$]$ ).

3. Main results. The peculiarities of the straight-line location of points in an arbitrary metric space are due to the use of the angle of equality (1) as a numerical characteristic. However, this choice makes it possible to establish a number of relationships that will be performed in any metric spaces, without restrictions on their metrics, and will have the corresponding geometric interpretation in Euclidean geometry. In particular, the following statements hold.

Lemma 1. If the for the three points $x_{1}, x_{2}, x_{3}$ of a space $\Pi \varphi_{123}=0$, then

$$
\rho_{13}^{2}=\rho_{12}^{2}+\rho_{23}^{2} .
$$


Proof. By formula (1) of the angular characteristics we have

$$
\varphi_{123}=\frac{\rho_{12}^{2}+\rho_{23}^{2}-\rho_{13}^{2}}{2 \rho_{12} \rho_{23}}=0 .
$$

This implies the equality $\rho_{12}^{2}+\rho_{23}^{2}-\rho_{13}^{2}=0$, or $\rho_{13}^{2}=\rho_{12}^{2}+\rho_{23}^{2}$.

This simple consequence of Definition 1 can be regarded as the extension of the Pythagorean theorem to an arbitrary metric space.

Theorem 2. If for four points $x_{1}, x_{2}, x_{3}, x_{4}$ of a space $\Pi \varphi_{123}=-1, \varphi_{124}=\varphi_{324}=\varphi_{143}=0$, then

$$
\rho_{24}^{2}=\rho_{12} \rho_{23} .
$$

Proof. From the equality $\varphi_{123}=-1$, by the formula of the angular characteristic, we obtain

$$
\varphi_{123}=\frac{\rho_{12}^{2}+\rho_{23}^{2}-\rho_{13}^{2}}{2 \rho_{12} \rho_{23}}=-1,
$$

or

$$
\begin{array}{cl}
\rho_{12}^{2}+\rho_{23}^{2}-\rho_{13}^{2}=-2 \rho_{12} \rho_{23}, & \rho_{12}^{2}+2 \rho_{12} \rho_{23}+\rho_{23}^{2}=\rho_{13}^{2}, \\
\left(\rho_{12}+\rho_{23}\right)^{2}=\rho_{13}^{2}, & \rho_{13}=\rho_{12}+\rho_{23} .
\end{array}
$$

In addition, the equalities $\varphi_{124}=\varphi_{324}=\varphi_{143}=0$, by Lemma 1 , imply the equalities

$$
\begin{gathered}
\rho_{14}^{2}=\rho_{12}^{2}+\rho_{24}^{2}, \quad \rho_{34}^{2}=\rho_{23}^{2}+\rho_{24}^{2}, \\
\rho_{13}^{2}=\rho_{14}^{2}+\rho_{34}^{2} .
\end{gathered}
$$

Using the equalities obtained above, we get

$$
\rho_{13}^{2}=\left(\rho_{12}+\rho_{23}\right)^{2}=\rho_{12}^{2}+2 \rho_{12} \rho_{23}+\rho_{23}^{2} .
$$

On the other hand,

$$
\rho_{13}^{2}=\rho_{14}^{2}+\rho_{34}^{2}=\left(\rho_{12}^{2}+\rho_{24}^{2}\right)+\left(\rho_{23}^{2}+\rho_{24}^{2}\right)=\rho_{12}^{2}+2 \rho_{24}^{2}+\rho_{23}^{2} .
$$

Equating the right-hand sides of both equalities we obtain equality (4).

Theorem 2 can be considered as an analogue for an arbitrary metric space of a well-known in Euclidean geometry statement, that the height that is held from a vertex of straight angle of triangle to the hypotenuse is the geometric mean for two segments on the which the height divides the hypotenuse. However, due to the peculiarity of adjacent angles in the space $\Pi$, in the condition of Theorem 2, unlike in the Euclidean geometry, it is necessary to require that both angles $\angle\left(x_{1}, x_{2}, x_{4}\right)$ and $\angle\left(x_{3}, x_{2}, x_{4}\right)$ are straight angles. This condition can be replaced by the condition of the planar placement of points $x_{1}, x_{2}, x_{3}, x_{4}$ in the space $\Pi[5$, p. 387].

Theorem 3. For arbitrary three points $x_{1}, x_{2}, x_{3}$ of the space $\Pi$, the following equality holds

$$
\rho_{13}=\rho_{12} \varphi_{213}+\rho_{23} \varphi_{132} .
$$

Proof. Using equality (1) transform the right-hand side of equality (5)

$$
\rho_{12} \varphi_{213}+\rho_{23} \varphi_{132}=\rho_{12} \frac{\rho_{12}^{2}+\rho_{13}^{2}-\rho_{23}^{2}}{2 \rho_{12} \rho_{13}}+\rho_{23} \frac{\rho_{13}^{2}+\rho_{23}^{2}-\rho_{12}^{2}}{2 \rho_{13} \rho_{23}}=
$$




$$
=\frac{\rho_{12}^{2}+\rho_{13}^{2}-\rho_{23}^{2}+\rho_{13}^{2}+\rho_{23}^{2}-\rho_{12}^{2}}{2 \rho_{13}}=\frac{2 \rho_{13}^{2}}{2 \rho_{13}}=\rho_{13} .
$$

Consequently, equality (5) holds, in particular, it holds in the case of a "degenerate" triangle.

Note that equality (5) is an analogue of the well-known "projection formula" in Euclidean geometry [19, p. 26].

Theorem 4. For arbitrary three different points $x_{1}, x_{2}, x_{3}$ of the space $\Pi$

$$
\frac{1}{\rho_{23}^{2}}\left|\begin{array}{cc}
1 & \varphi_{213} \\
\varphi_{213} & 1
\end{array}\right|=\frac{1}{\rho_{13}^{2}}\left|\begin{array}{cc}
1 & \varphi_{123} \\
\varphi_{123} & 1
\end{array}\right|=\frac{1}{\rho_{12}^{2}}\left|\begin{array}{cc}
1 & \varphi_{132} \\
\varphi_{132} & 1
\end{array}\right| .
$$

Proof. In equalities (6) we compute and transform, for example, the first determinant. We obtain

$$
\begin{gathered}
\left|\begin{array}{cc}
1 & \varphi_{213} \\
\varphi_{213} & 1
\end{array}\right|=1-\varphi_{213}^{2}=1-\left(\frac{\rho_{12}^{2}+\rho_{13}^{2}-\rho_{23}^{2}}{2 \rho_{12} \rho_{13}}\right)^{2}=1-\frac{\left(\rho_{12}^{2}+\rho_{13}^{2}-\rho_{23}^{2}\right)^{2}}{4 \rho_{12}^{2} \rho_{13}^{2}}= \\
\left.=\frac{1}{4 \rho_{12}^{2} \rho_{13}^{2}} 4 \rho_{12}^{2} \rho_{13}^{2}-\left(\rho_{12}^{2}+\rho_{13}^{2}-\rho_{23}^{2}\right)^{2}\right)= \\
=\frac{1}{4 \rho_{12}^{2} \rho_{13}^{2}}\left(4 \rho_{12}^{2} \rho_{13}^{2}-\rho_{12}^{4}-\rho_{13}^{4}-\rho_{23}^{4}-2 \rho_{12}^{2} \rho_{13}^{2}+2 \rho_{12}^{2} \rho_{23}^{2}+2 \rho_{13}^{2} \rho_{23}^{2}\right)= \\
=\frac{1}{4 \rho_{12}^{2} \rho_{13}^{2}}\left(2 \rho_{12}^{2} \rho_{13}^{2}+2 \rho_{12}^{2} \rho_{23}^{2}+2 \rho_{13}^{2} \rho_{23}^{2}-\rho_{12}^{4}-\rho_{13}^{4}-\rho_{23}^{4}\right)= \\
=\frac{1}{4 \rho_{12}^{2} \rho_{13}^{2}}\left(4 \rho_{12}^{2} \rho_{23}^{2}-\rho_{12}^{4}-\rho_{23}^{4}-\rho_{13}^{4}-2 \rho_{12}^{2} \rho_{23}^{2}+2 \rho_{12}^{2} \rho_{13}^{2}+2 \rho_{13}^{2} \rho_{23}^{2}\right)= \\
=\frac{\rho_{23}^{2}}{4 \rho_{12}^{2} \rho_{13}^{2} \rho_{23}^{2}}\left(4 \rho_{12}^{2} \rho_{23}^{2}-\left(\rho_{12}^{2}+\rho_{23}^{2}-\rho_{13}^{2}\right)^{2}\right)=\frac{\rho_{23}^{2}}{\rho_{13}^{2}}\left(1-\frac{\left(\rho_{12}^{2}+\rho_{23}^{2}-\rho_{13}^{2}\right)^{2}}{4 \rho_{12}^{2} \rho_{23}^{2}}\right)= \\
=\frac{\rho_{23}^{2}}{\rho_{13}^{2}}\left(1-\left(\frac{\rho_{12}^{2}+\rho_{23}^{2}-\rho_{13}^{2}}{2 \rho_{12} \rho_{23}}\right)^{2}\right)=\frac{\rho_{23}^{2}}{\rho_{13}^{2}}\left(1-\varphi_{123}^{2}\right)=\frac{\rho_{23}^{2}}{\rho_{13}^{2}}\left|\begin{array}{cc}
1 & \varphi_{123} \\
\varphi_{123} & 1
\end{array}\right| .
\end{gathered}
$$

This implies the equality

$$
\frac{1}{\rho_{23}^{2}}\left|\begin{array}{cc}
1 & \varphi_{213} \\
\varphi_{213} & 1
\end{array}\right|=\frac{1}{\rho_{13}^{2}}\left|\begin{array}{cc}
1 & \varphi_{123} \\
\varphi_{123} & 1
\end{array}\right| .
$$

Similarly to the previous one, we obtain the equality

$$
\frac{1}{\rho_{23}^{2}}\left|\begin{array}{cc}
1 & \varphi_{213} \\
\varphi_{213} & 1
\end{array}\right|=\frac{1}{\rho_{12}^{2}}\left|\begin{array}{cc}
1 & \varphi_{132} \\
\varphi_{132} & 1
\end{array}\right| .
$$

Equating both equalities, we obtain equalities (6).

Equalities (6) can be regarded as analogous to the sine theorem in Euclidean geometry for arbitrary metric space.

For arbitrary four points of the space $\Pi$, the following statement holds. 
Theorem 5. For arbitrary four points $x_{1}, x_{2}, x_{3}, x_{4}$ of the space $\Pi$

$$
\begin{gathered}
\frac{1}{\rho_{23}^{2} \rho_{24}^{2} \rho_{34}^{2}}\left|\begin{array}{ccc}
1 & \varphi_{213} & \varphi_{214} \\
\varphi_{213} & 1 & \varphi_{314} \\
\varphi_{214} & \varphi_{314} & 1
\end{array}\right|=\frac{1}{\rho_{13}^{2} \rho_{14}^{2} \rho_{34}^{2}}\left|\begin{array}{ccc}
1 & \varphi_{123} & \varphi_{124} \\
\varphi_{123} & 1 & \varphi_{324} \\
\varphi_{124} & \varphi_{324} & 1
\end{array}\right|= \\
=\frac{1}{\rho_{12}^{2} \rho_{14}^{2} \rho_{24}^{2}}\left|\begin{array}{ccc}
1 & \varphi_{132} & \varphi_{134} \\
\varphi_{132} & 1 & \varphi_{234} \\
\varphi_{134} & \varphi_{234} & 1
\end{array}\right|=\frac{1}{\rho_{12}^{2} \rho_{13}^{2} \rho_{23}^{2}}\left|\begin{array}{cccc}
1 & \varphi_{142} & \varphi_{143} \\
\varphi_{142} & 1 & \varphi_{243} \\
\varphi_{143} & \varphi_{243} & 1
\end{array}\right| .
\end{gathered}
$$

Proof. Using the corresponding values of the angular characteristics, we calculate, for example, the determinant

$$
\begin{aligned}
& \left|\begin{array}{ccc}
1 & \varphi_{213} & \varphi_{214} \\
\varphi_{213} & 1 & \varphi_{314} \\
\varphi_{214} & \varphi_{314} & 1
\end{array}\right|=1+2 \varphi_{213} \varphi_{214} \varphi_{314}-\varphi_{213}^{2}-\varphi_{214}^{2}-\varphi_{314}^{2}= \\
& =1+2 \frac{\rho_{12}^{2}+\rho_{13}^{2}-\rho_{23}^{2}}{2 \rho_{12} \rho_{13}} \cdot \frac{\rho_{12}^{2}+\rho_{14}^{2}-\rho_{24}^{2}}{2 \rho_{12} \rho_{14}} \cdot \frac{\rho_{13}^{2}+\rho_{14}^{2}-\rho_{34}^{2}}{2 \rho_{13} \rho_{14}}- \\
& -\left(\frac{\rho_{12}^{2}+\rho_{13}^{2}-\rho_{23}^{2}}{2 \rho_{12} \rho_{13}}\right)^{2}-\left(\frac{\rho_{12}^{2}+\rho_{14}^{2}-\rho_{24}^{2}}{2 \rho_{12} \rho_{14}}\right)^{2}-\left(\frac{\rho_{13}^{2}+\rho_{14}^{2}-\rho_{34}^{2}}{2 \rho_{13} \rho_{14}}\right)^{2}= \\
& =1+\frac{\left(\rho_{12}^{2}+\rho_{13}^{2}-\rho_{23}^{2}\right)\left(\rho_{12}^{2}+\rho_{14}^{2}-\rho_{24}^{2}\right)\left(\rho_{13}^{2}+\rho_{14}^{2}-\rho_{34}^{2}\right)}{4 \rho_{12}^{2} \rho_{13}^{2} \rho_{14}^{2}}- \\
& -\frac{\left(\rho_{12}^{2}+\rho_{13}^{2}-\rho_{23}^{2}\right)^{2}}{4 \rho_{12}^{2} \rho_{13}^{2}}-\frac{\left(\rho_{12}^{2}+\rho_{14}^{2}-\rho_{24}^{2}\right)^{2}}{4 \rho_{12}^{2} \rho_{14}^{2}}-\frac{\left(\rho_{13}^{2}+\rho_{14}^{2}-\rho_{34}^{2}\right)^{2}}{4 \rho_{13}^{2} \rho_{14}^{2}}= \\
& =\frac{1}{4 \rho_{12}^{2} \rho_{13}^{2} \rho_{14}^{2}}\left(4 \rho_{12}^{2} \rho_{13}^{2} \rho_{14}^{2}+\left(\rho_{12}^{2}+\rho_{13}^{2}-\rho_{23}^{2}\right)\left(\rho_{12}^{2}+\rho_{14}^{2}-\rho_{24}^{2}\right)\left(\rho_{13}^{2}+\rho_{14}^{2}-\rho_{34}^{2}\right)-\right. \\
& \left.-\rho_{14}^{2}\left(\rho_{12}^{2}+\rho_{13}^{2}-\rho_{23}^{2}\right)^{2}-\rho_{13}^{2}\left(\rho_{12}^{2}+\rho_{14}^{2}-\rho_{24}^{2}\right)^{2}-\rho_{12}^{2}\left(\rho_{13}^{2}+\rho_{14}^{2}-\rho_{34}^{2}\right)^{2}\right)= \\
& =\frac{1}{4 \rho_{12}^{2} \rho_{13}^{2} \rho_{14}^{2}}\left(4 \rho_{12}^{2} \rho_{13}^{2} \rho_{14}^{2}\right)+\left(\rho_{12}^{2}+\rho_{13}^{2}-\rho_{23}^{2}\right)\left(\rho_{12}^{2} \rho_{13}^{2}+\rho_{12}^{2} \rho_{14}^{2}-\rho_{12}^{2} \rho_{34}^{2}+\right. \\
& \left.+\rho_{13}^{2} \rho_{14}^{2}+\rho_{14}^{4}-\rho_{14}^{2} \rho_{34}^{2}-\rho_{13}^{2} \rho_{24}^{2}-\rho_{14}^{2} \rho_{24}^{2}+\rho_{24}^{2} \rho_{34}^{2}\right)- \\
& -\rho_{14}^{2}\left(\rho_{12}^{4}+\rho_{13}^{4}+\rho_{23}^{4}+2 \rho_{12}^{2} \rho_{13}^{2}-2 \rho_{12}^{2} \rho_{23}^{2}-2 \rho_{13}^{2} \rho_{23}^{2}\right)- \\
& -\rho_{13}^{2}\left(\rho_{12}^{4}+\rho_{14}^{4}+\rho_{24}^{4}+2 \rho_{12}^{2} \rho_{14}^{2}-2 \rho_{12}^{2} \rho_{24}^{2}-2 \rho_{14}^{2} \rho_{24}^{2}\right)- \\
& \left.-\rho_{12}^{2}\left(\rho_{13}^{4}+\rho_{14}^{4}+\rho_{34}^{4}+2 \rho_{13}^{2} \rho_{14}^{2}-2 \rho_{13}^{2} \rho_{34}^{2}-2 \rho_{14}^{2} \rho_{34}^{2}\right)\right)= \\
& =\frac{1}{4 \rho_{12}^{2} \rho_{13}^{2} \rho_{14}^{2}}\left(4 \rho_{12}^{2} \rho_{13}^{2} \rho_{14}^{2}+\rho_{12}^{4} \rho_{13}^{2}+\rho_{12}^{4} \rho_{14}^{2}-\rho_{12}^{4} \rho_{34}^{2}+\rho_{12}^{2} \rho_{13}^{2} \rho_{14}^{2}+\rho_{12}^{2} \rho_{14}^{4}-\right. \\
& -\rho_{12}^{2} \rho_{14}^{2} \rho_{34}^{2}-\rho_{12}^{2} \rho_{13}^{2} \rho_{24}^{2}-\rho_{12}^{2} \rho_{14}^{2} \rho_{24}^{2}+\rho_{12}^{2} \rho_{24}^{2} \rho_{34}^{2}+ \\
& +\rho_{12}^{2} \rho_{13}^{4}+\rho_{12}^{2} \rho_{13}^{2} \rho_{14}^{2}-\rho_{12}^{2} \rho_{13}^{2} \rho_{34}^{2}+\rho_{13}^{4} \rho_{14}^{2}+\rho_{13}^{2} \rho_{14}^{4}-\rho_{13}^{2} \rho_{14}^{2} \rho_{34}^{2}-\rho_{13}^{4} \rho_{24}^{2}- \\
& -\rho_{13}^{2} \rho_{14}^{2} \rho_{24}^{2}+\rho_{13}^{2} \rho_{24}^{2} \rho_{34}^{2}- \\
& -\rho_{12}^{2} \rho_{13}^{2} \rho_{23}^{2}-\rho_{12}^{2} \rho_{14}^{2} \rho_{23}^{2}+\rho_{12}^{2} \rho_{23}^{2} \rho_{34}^{2}-\rho_{13}^{2} \rho_{14}^{2} \rho_{23}^{2}-\rho_{14}^{4} \rho_{23}^{2}+\rho_{14}^{2} \rho_{23}^{2} \rho_{34}^{2}+ \\
& +\rho_{13}^{2} \rho_{23}^{2} \rho_{24}^{2}+\rho_{14}^{2} \rho_{23}^{2} \rho_{24}^{2}-\rho_{23}^{2} \rho_{24}^{2} \rho_{34}^{2}- \\
& -\rho_{12}^{4} \rho_{14}^{2}-\rho_{13}^{4} \rho_{14}^{2}-\rho_{14}^{2} \rho_{23}^{4}-2 \rho_{12}^{2} \rho_{13}^{2} \rho_{14}^{2}+2 \rho_{12}^{2} \rho_{14}^{2} \rho_{23}^{2}+2 \rho_{13}^{2} \rho_{14}^{2} \rho_{23}^{2}- \\
& -\rho_{12}^{4} \rho_{13}^{2}-\rho_{13}^{2} \rho_{14}^{4}-\rho_{13}^{2} \rho_{24}^{4}-2 \rho_{12}^{2} \rho_{13}^{2} \rho_{14}^{2}+2 \rho_{12}^{2} \rho_{13}^{2} \rho_{24}^{2}+2 \rho_{13}^{2} \rho_{14}^{2} \rho_{24}^{2}- \\
& \left.-\rho_{12}^{2} \rho_{13}^{4}-\rho_{12}^{2} \rho_{14}^{4}-\rho_{12}^{2} \rho_{34}^{4}-2 \rho_{12}^{2} \rho_{13}^{2} \rho_{14}^{2}+2 \rho_{12}^{2} \rho_{13}^{2} \rho_{34}^{2}+2 \rho_{12}^{2} \rho_{14}^{2} \rho_{34}^{2}\right)=
\end{aligned}
$$




$$
\begin{gathered}
=\frac{1}{4 \rho_{12}^{2} \rho_{13}^{2} \rho_{14}^{2}}\left(-\rho_{12}^{4} \rho_{34}^{2}-\rho_{12}^{2} \rho_{13}^{2} \rho_{23}^{2}+\rho_{12}^{2} \rho_{13}^{2} \rho_{24}^{2}+\rho_{12}^{2} \rho_{13}^{2} \rho_{34}^{2}+\rho_{12}^{2} \rho_{14}^{2} \rho_{23}^{2}-\rho_{12}^{2} \rho_{14}^{2} \rho_{24}^{2}+\right. \\
+\rho_{12}^{2} \rho_{14}^{2} \rho_{34}^{2}+\rho_{12}^{2} \rho_{23}^{2} \rho_{34}^{2}+\rho_{12}^{2} \rho_{24}^{2} \rho_{34}^{2}-\rho_{12}^{2} \rho_{34}^{4}- \\
-\rho_{13}^{4} \rho_{24}^{2}+\rho_{13}^{2} \rho_{14}^{2} \rho_{23}^{2}+\rho_{13}^{2} \rho_{14}^{2} \rho_{24}^{2}-\rho_{13}^{2} \rho_{14}^{2} \rho_{34}^{2}+\rho_{13}^{2} \rho_{23}^{2} \rho_{24}^{2}-\rho_{13}^{2} \rho_{24}^{4}+\rho_{13}^{2} \rho_{24}^{2} \rho_{34}^{2}- \\
\left.-\rho_{14}^{4} \rho_{23}^{2}-\rho_{14}^{2} \rho_{23}^{4}+\rho_{14}^{2} \rho_{23}^{2} \rho_{24}^{2}+\rho_{14}^{2} \rho_{23}^{2} \rho_{34}^{2}-\rho_{23}^{2} \rho_{24}^{2} \rho_{34}^{2}\right)= \\
=\frac{1}{4 \rho_{12}^{2} \rho_{13}^{2} \rho_{14}^{2}}\left(\rho_{12}^{2} \rho_{34}^{2}\left(\rho_{13}^{2}+\rho_{14}^{2}+\rho_{23}^{2}+\rho_{24}^{2}-\rho_{12}^{2}-\rho_{34}^{2}\right)+\right. \\
+\rho_{13}^{2} \rho_{24}^{2}\left(\rho_{12}^{2}+\rho_{14}^{2}+\rho_{23}^{2}+\rho_{34}^{2}-\rho_{13}^{2}-\rho_{24}^{2}\right)+\rho_{14}^{2} \rho_{23}^{2}\left(\rho_{12}^{2}+\rho_{13}^{2}+\rho_{24}^{2}+\rho_{34}^{2}-\rho_{14}^{2}-\rho_{23}^{2}\right)- \\
\left.-\rho_{12}^{2} \rho_{13}^{2} \rho_{23}^{2}-\rho_{12}^{2} \rho_{14}^{2} \rho_{24}^{2}-\rho_{13}^{2} \rho_{14}^{2} \rho_{34}^{2}-\rho_{23}^{2} \rho_{24}^{2} \rho_{34}^{2}\right)=\frac{1}{8 \rho_{12}^{2} \rho_{13}^{2} \rho_{14}^{2}} \Gamma\left(x_{1}, x_{2}, x_{3}, x_{4}\right),
\end{gathered}
$$

where $\Gamma\left(x_{1}, x_{2}, x_{3}, x_{4}\right)$ is the Cayley-Menger determinant for the four points $x_{1}, x_{2}, x_{3}, x_{4}$ of the space $\Pi([13$, p. 290])

$$
\begin{gathered}
\Gamma\left(x_{1}, x_{2}, x_{3}, x_{4}\right)=\left|\begin{array}{ccccc}
0 & 1 & 1 & 1 & 1 \\
1 & 0 & \rho_{12}^{2} & \rho_{13}^{2} & \rho_{14}^{2} \\
1 & \rho_{12}^{2} & 0 & \rho_{23}^{2} & \rho_{24}^{2} \\
1 & \rho_{13}^{2} & \rho_{23}^{2} & 0 & \rho_{34}^{2} \\
1 & \rho_{14}^{2} & \rho_{24}^{2} & \rho_{34}^{2} & 0
\end{array}\right|= \\
=2\left(\rho_{12}^{2} \rho_{34}^{2}\left(\rho_{13}^{2}+\rho_{14}^{2}+\rho_{23}^{2}+\rho_{24}^{2}-\rho_{12}^{2}-\rho_{34}^{2}\right)+\rho_{13}^{2} \rho_{24}^{2}\left(\rho_{12}^{2}+\rho_{14}^{2}+\rho_{23}^{2}+\rho_{34}^{2}-\rho_{13}^{2}-\rho_{24}^{2}\right)+\right. \\
\left.+\rho_{14}^{2} \rho_{23}^{2}\left(\rho_{12}^{2}+\rho_{13}^{2}+\rho_{24}^{2}+\rho_{34}^{2}-\rho_{14}^{2}-\rho_{23}^{2}\right)-\rho_{12}^{2} \rho_{13}^{2} \rho_{23}^{2}-\rho_{12}^{2} \rho_{14}^{2} \rho_{24}^{2}-\rho_{13}^{2} \rho_{14}^{2} \rho_{34}^{2}-\rho_{23}^{2} \rho_{24}^{2} \rho_{34}^{2}\right) .
\end{gathered}
$$

We multiply the two parts of the equality by the expression $\frac{1}{\rho_{23}^{2} \rho_{24}^{2} \rho_{34}^{2}}$ and obtain

$$
\frac{1}{\rho_{23}^{2} \rho_{24}^{2} \rho_{34}^{2}}\left|\begin{array}{ccc}
1 & \varphi_{213} & \varphi_{214} \\
\varphi_{213} & 1 & \varphi_{314} \\
\varphi_{214} & \varphi_{314} & 1
\end{array}\right|=\frac{1}{8 \rho_{12}^{2} \rho_{13}^{2} \rho_{14}^{2} \rho_{23}^{2} \rho_{24}^{2} \rho_{34}^{2}} \Gamma\left(x_{1}, x_{2}, x_{3}, x_{4}\right) .
$$

Similar to the previous one, we find

$$
\begin{gathered}
\frac{1}{8 \rho_{12}^{2} \rho_{13}^{2} \rho_{14}^{2} \rho_{23}^{2} \rho_{24}^{2} \rho_{34}^{2}} \Gamma\left(x_{1}, x_{2}, x_{3}, x_{4}\right)=\frac{1}{\rho_{13}^{2} \rho_{14}^{2} \rho_{34}^{2}}\left|\begin{array}{ccc}
1 & \varphi_{123} & \varphi_{124} \\
\varphi_{123} & 1 & \varphi_{324} \\
\varphi_{124} & \varphi_{324} & 1
\end{array}\right|= \\
=\frac{1}{\rho_{12}^{2} \rho_{14}^{2} \rho_{24}^{2}}\left|\begin{array}{ccc}
1 & \varphi_{132} & \varphi_{134} \\
\varphi_{132} & 1 & \varphi_{234} \\
\varphi_{134} & \varphi_{234} & 1
\end{array}\right|=\frac{1}{\rho_{12}^{2} \rho_{13}^{2} \rho_{23}^{2}}\left|\begin{array}{ccc}
1 & \varphi_{142} & \varphi_{143} \\
\varphi_{142} & 1 & \varphi_{243} \\
\varphi_{143} & \varphi_{243} & 1
\end{array}\right| .
\end{gathered}
$$

By equating these values, we obtain equality (7).

In the space $\mathbb{R}^{3}$ equalities (7) associate the plane angles each of the four vertices of the tetrahedron and the lengths of its edges. The known analogues of the sine theorem for a tetrahedron in the space $\mathbb{R}^{3}$ connect between themselves planar angles each of the four vertices of the tetrahedron, the lengths of its edges and the linear angles of the dihedral angles at each of these edges (the so-called C. Staudt sines [20, p. 99]). For the case of an arbitrary metric space with a finite or countable number of points, it is rather difficult to apply these formulas. 
Let us verify equalities (7) for an arbitrary tetrahedron in the space $\mathbb{R}^{3}$. In [21, p. 61], in the notation of this paper, for the space $\mathbb{R}^{3}$, the following equality is established

$$
\left|\begin{array}{ccc}
1 & \varphi_{213} & \varphi_{214} \\
\varphi_{213} & 1 & \varphi_{314} \\
\varphi_{214} & \varphi_{314} & 1
\end{array}\right|=\frac{36 V^{2}}{\rho_{12}^{2} \rho_{13}^{2} \rho_{14}^{2}}
$$

where $V$ is the volume of the tetrahedron with vertices at the points $x_{1}, x_{2}, x_{3}, x_{4}$. For the same tetrahedron, we obtain the equalities

$$
\begin{aligned}
& \left|\begin{array}{ccc}
1 & \varphi_{123} & \varphi_{124} \\
\varphi_{123} & 1 & \varphi_{324} \\
\varphi_{124} & \varphi_{324} & 1
\end{array}\right|=\frac{36 V^{2}}{\rho_{12}^{2} \rho_{23}^{2} \rho_{24}^{2}}, \quad\left|\begin{array}{ccc}
1 & \varphi_{132} & \varphi_{134} \\
\varphi_{132} & 1 & \varphi_{234} \\
\varphi_{134} & \varphi_{234} & 1
\end{array}\right|=\frac{36 V^{2}}{\rho_{13}^{2} \rho_{23}^{2} \rho_{34}^{2}}, \\
& \left|\begin{array}{ccc}
1 & \varphi_{142} & \varphi_{143} \\
\varphi_{142} & 1 & \varphi_{243} \\
\varphi_{143} & \varphi_{243} & 1
\end{array}\right|=\frac{36 V^{2}}{\rho_{14}^{2} \rho_{24}^{2} \rho_{34}^{2}} .
\end{aligned}
$$

Substituting these values of the determinants in equality (7) we obtain

$$
\begin{gathered}
\quad \frac{1}{\rho_{23}^{2} \rho_{24}^{2} \rho_{34}^{2}}\left|\begin{array}{cccc}
1 & \varphi_{213} & \varphi_{214} \\
\varphi_{213} & 1 & \varphi_{314} \\
\varphi_{214} & \varphi_{314} & 1
\end{array}\right|=\frac{1}{\rho_{13}^{2} \rho_{14}^{2} \rho_{34}^{2}}\left|\begin{array}{ccc}
1 & \varphi_{123} & \varphi_{124} \\
\varphi_{123} & 1 & \varphi_{324} \\
\varphi_{124} & \varphi_{324} & 1
\end{array}\right|= \\
=\frac{1}{\rho_{12}^{2} \rho_{14}^{2} \rho_{24}^{2}}\left|\begin{array}{cccc}
1 & \varphi_{132} & \varphi_{134} \\
\varphi_{132} & 1 & \varphi_{234} \\
\varphi_{134} & \varphi_{234} & 1
\end{array}\right|=\frac{1}{\rho_{12}^{2} \rho_{13}^{2} \rho_{23}^{2}}\left|\begin{array}{ccc}
1 & \varphi_{142} & \varphi_{143} \\
\varphi_{142} & 1 & \varphi_{243} \\
\varphi_{143} & \varphi_{243} & 1
\end{array}\right|==\frac{36 V^{2}}{\rho_{12}^{2} \rho_{13}^{2} \rho_{14}^{2} \rho_{23}^{2} \rho_{24}^{2} \rho_{34}^{2}} .
\end{gathered}
$$

The resulting expression in the space $\mathbb{R}^{3}$ is constant for a given tetrahedron, therefore equalities (7) are valid in this space.

\section{REFERENCES}

1. Kuz'mich V.I., The concept of angle in the study of the properties of a metric space, Visnyk Cherkaskoho Univ. Ser.: Ped. Nauky, 13 (2016), 26-32. (in Ukrainian)

2. Kuz'mich V.I., Angular characteristic in metric space, Algebr. and Geom. Methods of Analysis: Int. sci. Conf.: Abstracts, 2017, 11-12. (in Ukrainian)

3. Kuz'mich V.I., Flat placement set of points in a metric space, Visnyk Lviv Univ. Ser.: Mech.-Math., 83 (2017), 58-71. (in Ukrainian)

4. Kuz'mich V.I., Construction of flat images in an arbitrary metric space, Visnyk Cherkaskoho Univ. Ser.: Ped. Nauky, 11 (2017), 40-46. (in Ukrainian)

5. Kuz'mich V.I., Geometric properties of metric spaces, Ukr. Math. J., 71 (2019), №3, 382-399. (in Ukrainian)

6. Kagan V.F., The foundations of geometry. Part 2, M.-L.: Gostehizdat, 1956. (in Russian)

7. Kagan V.F., Essays on geometry, M.: Izdatel'stvo Moskovskogo universiteta, 1963. (in Russian)

8. Euclid, Elements. Books I-VI, M.-L.: Gostehizdat, 1948. (in Russian)

9. Hadamard J., Elementary geometry. Part 1, M.-L.: Gostehizdat, 1948. (in Russian)

10. Hilbert D., The foundations of geometry, Petrograd: Seyatel, 1923. (in Russian)

11. Aleksandrov A.D., Intrinsic geometry of convex surfaces, M.-L.: Hostehizdat, 1948. (in Russian)

12. Burago D., Burago Y., Ivanov S., A course in metric geometry, AMS: Providence, Rhode Island, 2001. 
13. Berger M., Geometrie. Part 1, M.: Mir, 1984.

14. Ponarin Ya.P., Elementary geometry. Part 1, M.: Moscow Center for Continuous Math. Educ., 2004. (in Russian)

15. Ponarin Ya.P., Elementary geometry. Part 2, M.: Moscow Center for Continuous Math. Educ., 2006. (in Russian)

16. Kuz'mich V.I., Kuz'mich Yu.V. Analogs of formula Jungius volume tetrahedron, Visnyk Cherkaskoho Univ. Ser.: Ped. Nauky, 36(249) (2012), 55-64. (in Ukrainian)

Kherson State University, Kherson, Ukraine

vikuzmichksu@gmail.com

Kherson State Agrarian University, Kherson, Ukraine

savchenko.o.g@ukr.net

Received 19.07.2019 